\title{
PRELIMINARY RESULTS FROM THE NRL/ATM INSTRUMENTS FROM SKYLAB SL/2
}

R. TOUSEY, J.-D. F. BARTOE, J. D. BOHLIN, G. E. BRUECKNER, J. D. PURCELL, V. E. SCHERRER, R. J. SCHUMACHER, N. R. SHEELEY, and M. E. VANHOOSIER U.S. Naval Research Laboratory, Washington, D.C. 20375, U.S.A.

\begin{abstract}
The Naval Research Laboratory prepared three instruments for ATM and also a series of rocket payloads for purposes of calibration, under the overall direction of R. Tousey, the Principal Investigator. In this preliminary report a summary of results from operations during the first mission (SL/2) and sample results are presented.

The extreme ultraviolet (XUV) spectroheliograph, S082A, performed well, obtaining 219 exposures over the two ranges, $150-350 \AA$ and $300-630 \AA$ with spatial resolution reaching at least $2^{\prime \prime}$ near the grating normal. Figure 1 , left to right upper, is a section from $200-304 \AA$; below, $304-400 \AA$ is covered. The exposures were made during an M-2 flare on June 15, 1973, which produced the conspicuous linear array
\end{abstract}

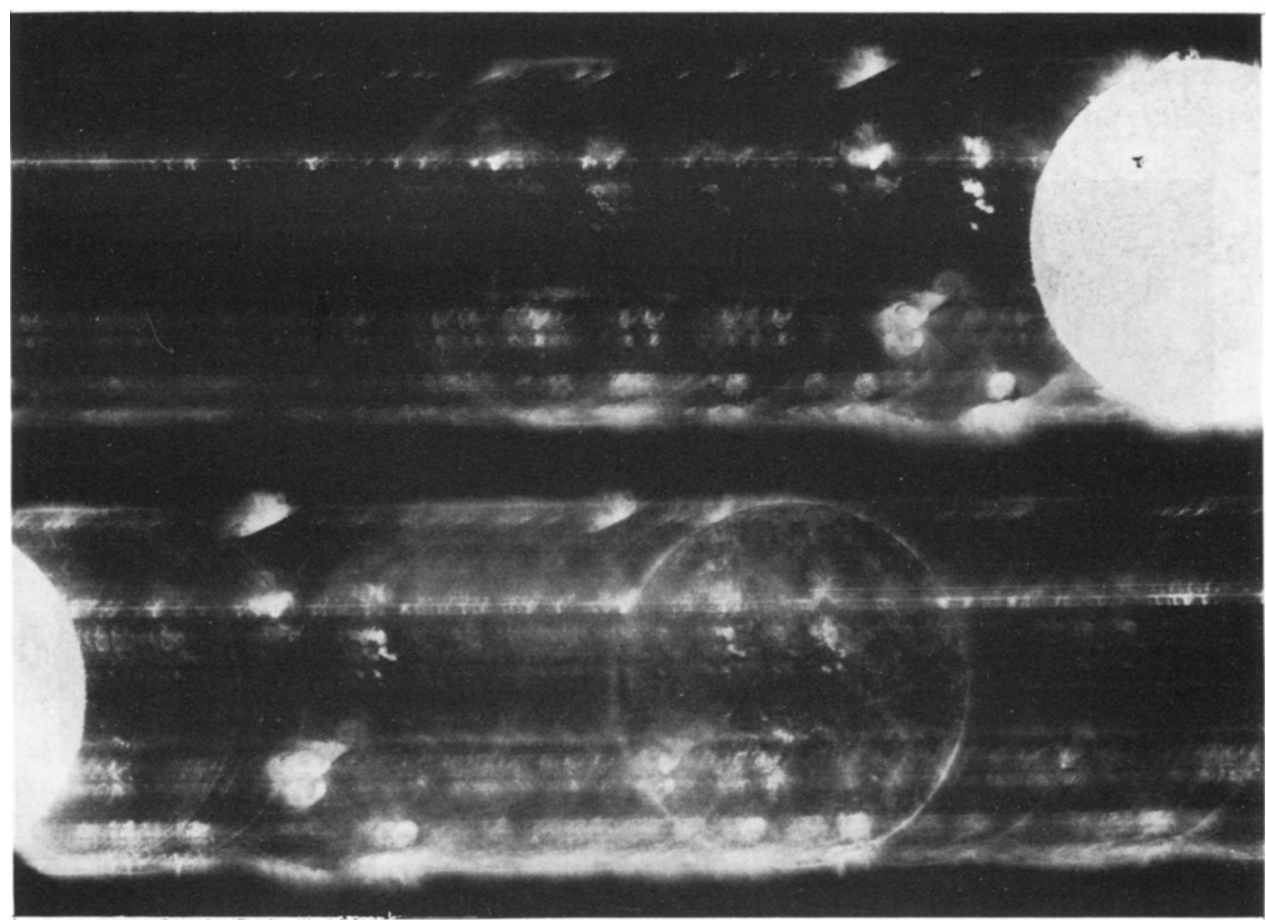

Fig. 1. Spectroheliograms of the $200-400 \AA$ region obtained by S082A on June 15,1973 during an M-2 flare. In the upper section the flare is seen in He II $304 \AA$ as the solarized black feature against the disk, followed by images converging to the series limit at $228 \AA$ and the continuum. In the lower section the flare is conspicuous in Fe XVI $335 \AA$ and $361 \AA$. Solar north is left and west up. $\lambda$ increases to the right. 
of images. In He II, the flare in $304 \AA$ is black by solarization against the overexposed image of the disk. Thirteen series members are resolved, followed by the continuum beyond $228 \AA$. Present are flare images in many highly ionized species such as Fe XIV, $\mathrm{XV}$, and XVI. In all these images the flare is complex in form, containing ribbons and threads. A few extremely high ionization lines were recorded, and in these the form was a nucleus without ribbons. In Figure $1 \mathrm{Fe}$ xxIV $255 \AA$ is present as the nearly circular feature about $30^{\prime \prime}$ in diameter, $\frac{1}{3}$ to the right across the upper spectrum.

Many other striking features are present. The active regions show loops and swirls that appear to delineate magnetic field lines in the corona. Fine detail as well as diffuse clouds are present, seen both against the disc and above the limb. Fe XV $284 \AA$ shows these forms beautifully.

The quiet disk in coronal lines is dark, but shows diffuse emission above the limb with a darker disc below where activity is present, and intense localized emission above active regions. In transition layer and low coronal lines the limb is a bright ring. $\mathrm{Mg}$ IX $368 \AA$ produces the intense ring in the lower part of Figure 1. The limb is not uniform, but contains breaks, points of origin of loops, and varies in width, depending on the character of the surface nearby.

Spicules are conspicuous in He II $304 \AA$, especially over the polar regions where the surface is rather dark. In Figure 1 few quiescent prominences are present in $\mathrm{He}$ II, but most of the emission high above the limb is Si XI.

A properly exposed He II $304 \AA$ disc image in Figure 2 is compared with an image

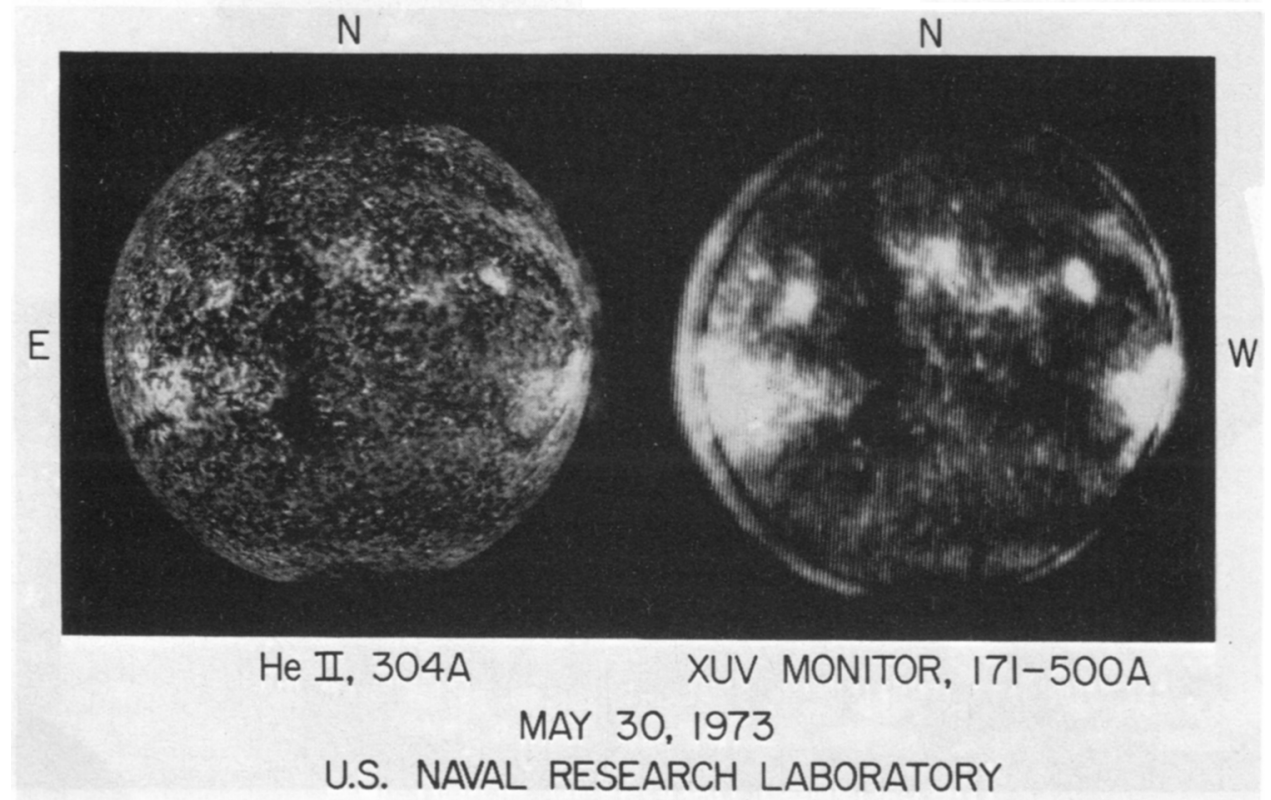

Fig. 2. An S082A spectroheliograph in He II $304 \AA$ compared with a heliogram, (171-500 $\AA$ ), produced by TV by the NRL XUV Monitor. Both images were made on May 30, 1973 and are mounted with north up and east left. 
produced by the NRL 'XUV Monitor'. This projected an undispersed image, $80 \%$ coronal and $20 \%$ chromospheric, from $171-500 \AA$, on a phosphor conversion layer deposited on the face plate of an SEC Vidicon. This TV image, viewed by the crewman and also transmitted to ground, had about 10-15" spatial resolution, and by means of a reticle array could be used for pointing the ATM. In He II the chromospheric network is present almost everywhere, but varies in character with boundaries crisp in some regions, and diffuse in others. The regions of diffuse network seem to correspond to regions that are mainly unipolar, such as regions on each side of a filament channel.

There are also regions where the emission is depressed. In Figure 2 there are five: one at each pole; the great rift running from the N-polar hole to $30^{\circ} \mathrm{S}$; a broad, long region following latitude $45^{\circ} \mathrm{S}$ which is the remnant of a filament channel; and another filament channel complex in the NW. The darkest of these are now called coronal holes, but their presence in $\mathrm{He}$ II $304 \AA$, (and also in He I $584 \AA$ ) shows that they extend well down into the chromosphere.

Scattered over the He II image and seen best within the holes are many isolated bright points. These show also in undispersed XUV, and some are visible in the XUV Monitor image in spite of its low resolution. These points seem to be associated with small bipolar regions, or small unipolar regions in a region of opposite polarity.

The emission from the coronal lines shows well in the XUV Monitor, as emission high above the limb produced by intense active regions often behind the limb, and as intense emission from active regions on the disc. By contrast, in He II the emission over the limb is from prominences, plus coronal emission from the near-blend Si XI 303.32 $\AA$. Also, the active regions are less intense in He II.

More often than expected, eruptive prominences were recorded in He II $304 \AA$. Figure 3 shows the largest observed on SL/2. It was followed about $8 \mathrm{~h}$ later by an eruption recorded by the white-light coronagraph, presumably the result of another prominence near the same location that erupted later. No corresponding emission is visible in Fe XV $284 \AA$, or in the Si XI corona, which is seen clearly separated from $\mathrm{He}$ II by its dark limb. Although a detailed comparison with $\mathrm{H} \alpha$ has not yet been made, the $\mathrm{He}$ II eruptive prominence seems more intense and larger than those usually seen in $\mathrm{H} \alpha$.

The other major NRL instrument was the chromospheric UV spectrograph, S082B. The ranges covered were $970-1970 \AA$, and $1940-3940 \AA$, with spectral resolutions of 0.04 and $0.08 \AA$, respectively. The instrument was not stigmatic, but produced a slit spectrum from a region on the Sun $2^{\prime \prime} \times 60^{\prime \prime}$. The position and angle of the slit were controlled precisely by the crewman who used the white-light S082B slit display, the $\mathrm{H} \alpha$ display, or solar coordinates. The one camera carried on SL/2 recorded nearly 1600 spectra, and the three more cameras to be flown on SL/ 3 and 4 are expected to bring the total close to the limit, 4800 .

At this time, study of the SL/2 spectra has hardly begun. In Figure 4 short sections of two exposures are reproduced with slit tangent to the limb and 12" inside, or 4" above. The photospheric spectrum contains mainly Fraunhofer lines, but does show 


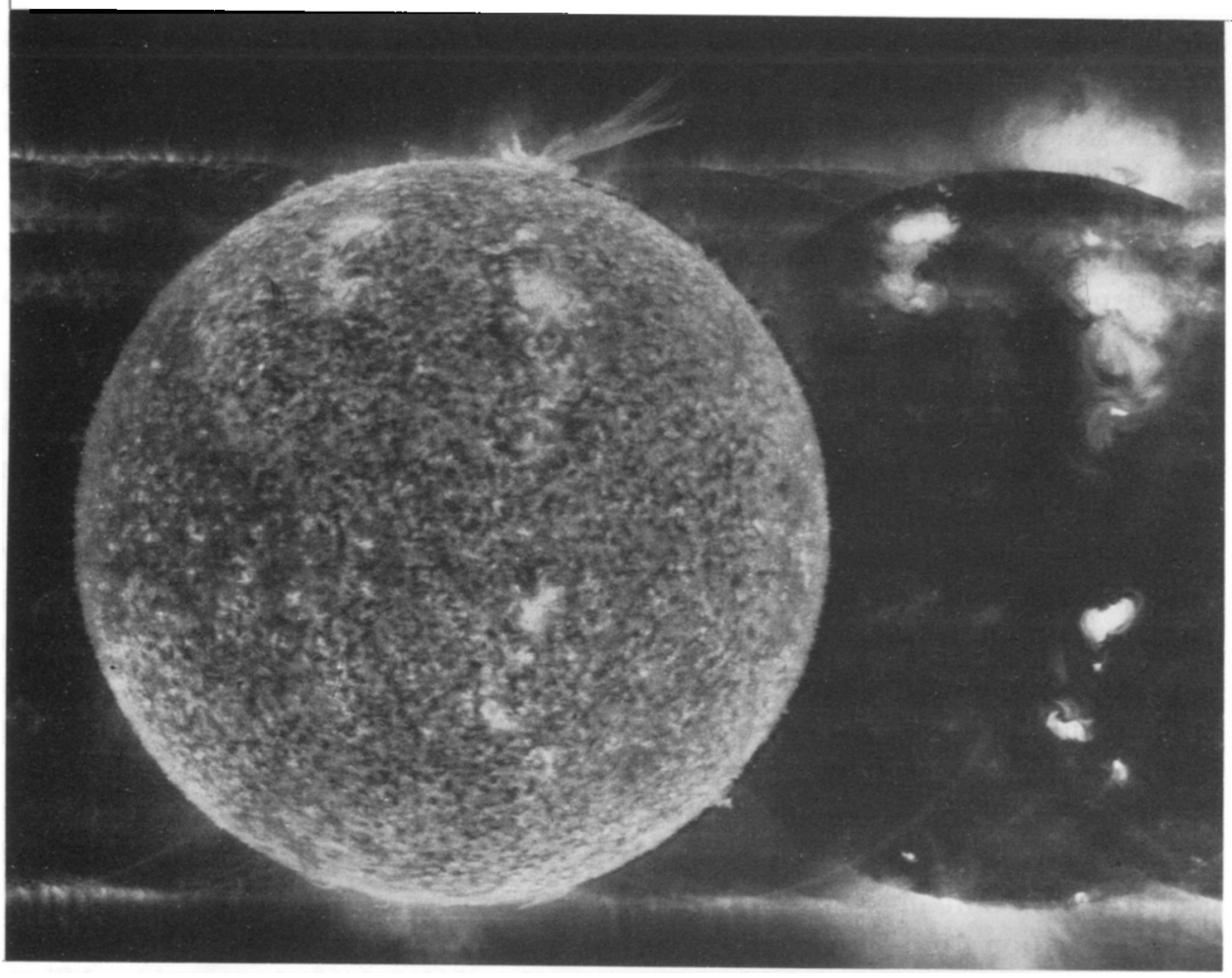

Fig. 3. A section of an S082A spectroheliogram showing He In $304 \AA$ with an eruptive prominence at $01: 46$ UT on June 10, 1973. The overlapping image to the right is Fe xv $284 \AA$.
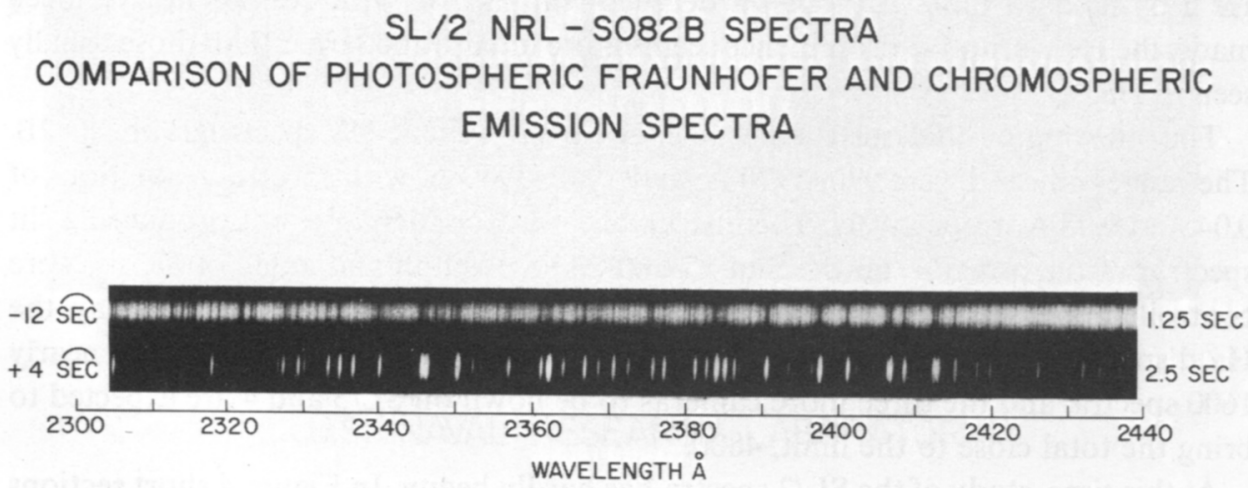

Fig. 4. A sample of spectra produced by S082B. Shown are short sections of spectra exposed with the $2^{\prime \prime} \times 60^{\prime \prime}$ slit placed $12^{\prime \prime}$ inside and $4^{\prime \prime}$ outside the limb. 
two emission lines of $\mathrm{Si}$ II, 2334.50, $2350.28 \AA$. Above the limb the photospheric continuum is absent, and the spectrum is chromospheric essentially like flash spectra recorded during a total eclipse. The instrument was used in major programs to record spectra across the limb, of prominences and filaments, active regions, the chromospheric network, and transients. Flare spectra were obtained. Another application was to photograph the setting sun to search for atmospheric contaminants and to measure the distribution of the normal atmospheric constituents to very high altitudes. The spectral resolution was sufficient to record line profiles and the results from $\mathrm{SL} / 2$ show a wealth of surprising shapes. 